\title{
Conformational Properties of an Adsorbed Charged Polymer
}

\author{
Chi-Ho Cheng ${ }^{1,2 *}$ and Pik-Yin Lai ${ }^{1,3}$ \\ ${ }^{1}$ Department of Physics and Center for Complex Systems, \\ National Central University, Taiwan \\ ${ }^{2}$ Institute of Physics, Academia Sinica, Taiwan \\ ${ }^{3}$ Physics Division, National Center for Theoretical Sciences, Taiwan
}

(Dated: November 4, 2018)

\begin{abstract}
The behavior of a strongly charged polymer adsorbed on an oppositely charged surface of lowdielectric constant is formulated by the functional integral method. By separating the translational, conformational, and fluctuational degrees of freedom, the scaling behaviors for both the height of the polymer and the thickness of the diffusion layer are determined. Unlike the results predicted by scaling theory, we identified the continuous crossover from the weak compression to the compression regime. All the analytical results are found to be consistent with Monte-Carlo simulations. Finally, an alternative (operational) definition of a charged polymer adsorption is proposed.
\end{abstract}

PACS numbers: $61.25 . \mathrm{Hq}, 82.35 . \mathrm{Gh}$

Charged polymer (polyelectrolyte) adsorption on charged surface remains an interesting and important problem due to its influence to material science [1], colloidal science [2], and biological science [3]. Hard substrates, and soft surfactant layers at interfaces can also be charged, due to the dissociation of ionic groups on the surfaces. Because the electrostatic force is strong and long-ranged, the electrostatic interaction between a charged polymer and a charged surface usually dominates over other non-electrostatic ones.

The problem of charged polymer adsorption on charged surface can be studied by many approaches [4]. By replacing the counterion effect by the Debye-Hückel potential within the linear mean-field theory, one solve the Edwards equation [5, 6, 7]. One can also solve both the Edwards equation and the Poisson-Boltzmann equation self-consistently 8, 9, 10] at nonlinear mean-field level in which the effective screening length near the charged surface may not be equal to the bulk one. Scaling theory were also applied to the problem 11]. Even more, the effect of attractive image forces from high-dielectric substrate 12, 13, 14], and repulsive image forces from low-dielectric substrate [15, 16, 17, 18] were also investigated by analytical methods or Monte-Carlo (MC) simulation.

However, the analytical approaches involving the Edwards equation usually impose zero monomer density at the charged surface in which the electrostatic boundary condition cannot be faithfully respected. It is only for the case of charged polymer adsorption on the highdielectric substrate studied by Cheng et al. 13] that the surface monomer density is properly treated. The surface monomer density follows a linear relation with surface charge density at Debye-Hückel level. It indicates that the charged polymer is fully compressed on the highdielectric substrate without any conformational change. For the low-dielectric substrate, due to the repulsive image forces, the polymer is not necessarily compressed on the substrate. Instead, the conformational degree of freedom plays an important role on the adsorption behavior.

In this paper, we study the conformational properties of charged polymer adsorbed on the low-dielectric substrate at Debye-Hückel level by both the functional integral methods and MC simulation. It is found that the usual Edwards equation is no longer valid to describe the non-Gaussian feature of polymer conformation. A new formulation by the functional integral method is proposed and compared with simulation results. Finally we give an operational definition of charged polymer adsorption.

A charged polymer carrying positive charges is immersed in a medium $(z>0)$ of dielectric constant $\epsilon$. At $z=0$ there is an impenetrable surface. Below the surface $(z<0)$, there is the substrate of low dielectric constant $\epsilon^{\prime}<\epsilon$. Just above the substrate, there is an uniform surface charge density $\sigma<0$. The adsorbed charged polymer always stays above the surface charge layer. Denote the charge on a polymer segment $d s$ by $q d s$, the Hamiltonian is

$$
\begin{aligned}
\mathcal{H}= & \frac{1}{2} \int_{0}^{N} d s \int_{0}^{N} d s^{\prime}\left(\Gamma \frac{\mathrm{e}^{-\kappa\left|\vec{r}(s)-\vec{r}\left(s^{\prime}\right)\right|}}{\left|\vec{r}(s)-\vec{r}\left(s^{\prime}\right)\right|}+\Gamma^{\prime}\left(2-\delta_{s, s^{\prime}}\right)\right. \\
& \left.\times \frac{\mathrm{e}^{-\kappa\left|\vec{r}(s)-\vec{r}^{\prime}\left(s^{\prime}\right)\right|}}{\left|\vec{r}(s)-\vec{r}^{\prime}\left(s^{\prime}\right)\right|}\right)-h \int_{0}^{N} d s \kappa^{-1} \mathrm{e}^{-\kappa \vec{r}(s) \cdot \hat{z}}
\end{aligned}
$$

where $s$ is the variable to parametrize the chain and $\kappa^{-1}$ the Debye screening length. $\vec{r}(s)=(x(s), y(s), z(s))$, $\vec{r}^{\prime}\left(s^{\prime}\right)=\left(x\left(s^{\prime}\right), y\left(s^{\prime}\right),-z\left(s^{\prime}\right)\right)$ are the positions of the monomers and their electrostatic images, respectively. $\Gamma=q^{2} / \epsilon, \Gamma^{\prime}=\Gamma\left(\epsilon-\epsilon^{\prime}\right) /\left(\epsilon+\epsilon^{\prime}\right)>0$, and $h=$ $4 \pi q|\sigma| /\left(\epsilon^{\prime}+\epsilon\right)>0$ are the coupling parameters governing the strengths of Coulomb interactions among the monomers themselves, between the polymer and its image, and between the polymer and the charged surface, respectively. Note that the above Hamiltonian is not exact even at Debye-Hückel level. In particular, the longi- 
tudinal interaction decays algebraically rather than exponentially [17]. However, the conformational properties related to the adsorption behavior will not be affected. We shall focus on the case of a charged polymer adsorption in a low ionic strength medium.

The continuum Hamiltonian in Eq. (1) is discretized to perform MC simulation. The continuous curve $\vec{r}(s)$ is replaced by a chain of beads $\vec{r}_{i}(i=1, \ldots, N)$ with hardcore excluded volume of finite radius $a$. The length and energy units are $2 a$ and $q^{2} / 2 \epsilon a$, respectively. Runs up to $10^{9} \mathrm{MC}$ steps and up to $N=120$ are performed.

The partition function of the system is

$$
\begin{aligned}
Z= & \int \mathcal{D}[\vec{r}(s)] \exp \left[-\frac{3}{2 a^{2}} \int_{0}^{N} d s\left(\frac{\partial \vec{r}(s)}{\partial s}\right)^{2}-\beta \mathcal{H}\right] \\
= & \int \prod_{i=1}^{N} d\left(\Delta \vec{r}_{i}\right) \exp \left[-\frac{3}{2 a^{2}} \sum_{i=1}^{N}\left(\Delta \vec{r}_{i}\right)^{2}-\beta \mathcal{H}\right] \\
= & \int \prod_{i=1}^{N} d\left(\Delta \vec{r}_{\| i}\right) d\left(\Delta z_{i}\right) \exp \left[-\frac{3}{2 a^{2}} \sum_{i=1}^{N}\left(\left(\Delta \vec{r}_{\| i}\right)^{2}+\left(\Delta z_{i}\right)^{2}\right)\right] \\
& \times \exp [-\beta \mathcal{H}]
\end{aligned}
$$

where $\vec{r}_{\|}(s)=(x(s), y(s))$ is the $x y$-plane projection of the curve $\vec{r}(s)$. While the charged polymer is adsorbed, $\left|\Delta z_{i}\right| \ll\left|\Delta \vec{r}_{\| i}\right|$, and note that $\vec{r}_{\|}(s)$ should describe a 2D polymer conformation. Hence we approximate

$$
\begin{aligned}
Z \simeq & \int \prod_{i=1}^{N} d\left(\Delta \vec{r}_{\| i}\right) d\left(\Delta z_{i}\right) \exp \left[-\frac{1}{a^{2}} \sum_{i=1}^{N}\left(\Delta \vec{r}_{\| i}\right)^{2}\right] \\
& \times \exp \left[-\frac{1}{2 a^{2}} \sum_{i=1}^{N}\left(\Delta \vec{r}_{\| i}\right)^{2}+\left(\Delta z_{i}\right)^{2}\right] \exp [-\beta \mathcal{H}] \\
= & \int \mathcal{D}\left[\vec{r}_{\|}(s), \vec{r}_{\perp}(s)\right] \exp \left[-\frac{1}{a^{2}} \int_{0}^{N} d s\left(\frac{\partial \vec{r}_{\|}(s)}{\partial s}\right)^{2}\right. \\
& \left.-\frac{1}{2 a^{2}} \int_{0}^{N} d s\left(\frac{\partial \vec{r}_{\perp}(s)}{\partial s}\right)^{2}-\beta \mathcal{H}\right]
\end{aligned}
$$

where $\vec{r}_{\perp}(s)=\left(\left|\vec{r}_{\|}(s)\right|, z(s)\right)$ is the side-view of $\vec{r}(s)$ along the curve $\vec{r}_{\|}(s)$. Note that the coefficients of the entropy terms of $\vec{r}_{\|}(s)$ and $\vec{r}_{\perp}(s)$ are $-1 / a^{2}$ and $-1 / 2 a^{2}$, respectively, which are different from that of $\vec{r}(s),-3 / 2 a^{2}$.

For the case of charged polymer adsorption, the selfelectrostatic interaction takes almost no effect in $\vec{r}_{\perp}(s)$ since $\left|\vec{r}(s)-\vec{r}\left(s^{\prime}\right)\right| \simeq\left|\vec{r}_{\|}(s)-\vec{r}_{\|}\left(s^{\prime}\right)\right|$. The repulsion from the images of the monomers can be effectively approximated by the interaction between each monomer and its image only. The residual repulsion is absorbed by renormalizing $\Gamma^{\prime}$. Then the partition function becomes

$$
\begin{aligned}
Z \simeq & \int \mathcal{D}\left[\vec{r}_{\|}(s)\right] \exp \left[-\frac{1}{a^{2}} \int_{0}^{N} d s\left(\frac{\partial \vec{r}_{\|}(s)}{\partial s}\right)^{2}\right. \\
& \left.-\frac{\beta \Gamma}{2} \int_{0}^{N} d s \int_{0}^{N} d s^{\prime} \frac{\mathrm{e}^{-\kappa\left|\vec{r}_{\|}(s)-\vec{r}_{\|}\left(s^{\prime}\right)\right|}}{\left|\vec{r}_{\|}(s)-\vec{r}_{\|}\left(s^{\prime}\right)\right|}\right]
\end{aligned}
$$

$$
\begin{aligned}
& \times \int \mathcal{D}\left[\vec{r}_{\perp}(s)\right] \exp \left[\int _ { 0 } ^ { N } d s \left\{-\frac{1}{2 a^{2}}\left(\frac{\partial \vec{r}_{\perp}(s)}{\partial s}\right)^{2}\right.\right. \\
& \left.\left.-\frac{\beta \Gamma^{\prime}}{4} \frac{\mathrm{e}^{-2 \kappa \vec{r}_{\perp}(s) \cdot \hat{z}}}{\vec{r}_{\perp}(s) \cdot \hat{z}}+\beta h \kappa^{-1} \mathrm{e}^{-\kappa \vec{r}_{\perp}(s) \cdot \hat{z}}\right\}\right]
\end{aligned}
$$

The system is decoupled into two independent degrees of freedom, $\vec{r}_{\|}(s)$ and $\vec{r}_{\perp}(s)$. Since the above functional integral with respect to $\vec{r}_{\|}(s)$ does not affect the adsorption behavior, we investigate only the conformational properties of $\vec{r}_{\perp}(s)$ in the following.

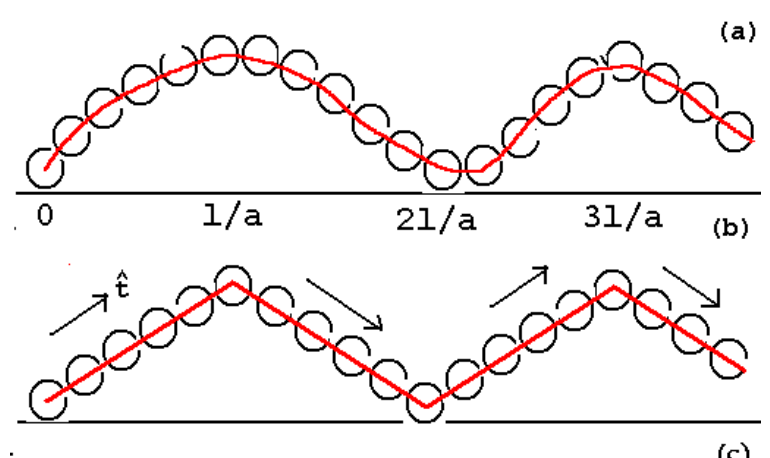

(c)

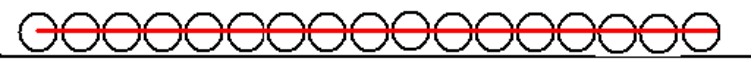

FIG. 1: Schematic diagram for the conformation of an adsorbed charged polymer. The degrees of freedom (entropies) of the polymer consists of three parts, the translation $\left(\vec{r}_{\perp \mathrm{c}}\right)$, the conformation $(\vec{t}(s))$, and the local fluctuation $\left(\delta \vec{r}_{\perp}(s)\right)$. The solid line represents the polymer orientation. The local fluctuation lies within the blobs. (a) The weakly compressed polymer (onset of adsorption) conformation in general. (b) The weakly compressed polymer conformation in our analytical approximation. (c) The compressed polymer (adsorption) conformation in which the conformational degree of freedom $\vec{t}(s)$ vanishes.

Because of the repulsive image force from the lowdielectric substrate, the charged polymer may be at weak compression or compression in which their schematic diagrams are shown in Fig प and प, respectively. The terminology of weak compression (onset of adsorption) and compression (adsorption) are borrowed from Borisov et al. 15, 16] for grafted polymers.

In order to distinguish between the weak compression and the compression in our formulation, and note that a slowly varying orientation of polymer conformation under weak compression, we decompose

$$
\vec{r}_{\perp}(s)=\vec{r}_{\perp \mathrm{c}}+\vec{t}(s)+\delta \vec{r}_{\perp}(s)
$$

where $\vec{r}_{\perp \mathrm{c}}=\frac{1}{N} \int_{0}^{N} d s \vec{r}_{\perp}(s)$ is the position of the center of mass, and $\vec{t}(s)$ is the orientation vector of the charged polymer. We also restrict

$$
\vec{t}(s) \cdot \delta \vec{r}_{\perp}(s)=0
$$


so that $\delta \vec{r}_{\perp}(s)$ represents the local fluctutation along $\vec{t}(s)$.

The adsorbed polymer is now characterized by translational $\left(\vec{r}_{\perp \mathrm{c}}\right)$, conformational $(\vec{t}(s))$, and local fluctutational $\left(\delta \vec{r}_{\perp}(s)\right)$ degrees of freedom. Under the compression regime, $\vec{t}(s)$ vanishes.

In general, it is hard to compute the effect from $\vec{t}(s)$. For simplicity but still capturing the qualitative picture of the weak compression as shown in Fig पa, we further make an approximation that

$$
\vec{t}(s) \cdot \hat{z}=\left\{\begin{array}{cc}
(2 a s / l-1) \vec{r}_{\perp c} \cdot \hat{z}, & 0<s<l / a \\
-(3-2 a s / l) \vec{r}_{\perp c} \cdot \hat{z}, & l / a<s<2 l / a
\end{array}\right.
$$

and repeat for a period of $2 l / a$. Its schematic diagram is shown in Fig 1). Substituting Eqs.(5)-(7) into Eq.(4), and at low-salt limit, we get

$$
\begin{aligned}
Z= & \mathcal{N}^{-1} \int d \vec{r}_{\perp \mathrm{c}} \exp \left[N \beta h \vec{r}_{\perp \mathrm{c}} \cdot \hat{z}\right] \int \mathcal{D}\left[\delta \vec{r}_{\perp}(s)\right] \\
& \exp \left[\int_{0}^{N} d s\left\{-\frac{1}{2 a^{2}}\left(\frac{\partial \delta \vec{r}_{\perp}(s)}{\partial s}\right)^{2}-\beta h \delta \vec{r}_{\perp}(s) \cdot \hat{z}\right\}\right] \\
& \times \int \mathcal{D}[\vec{t}(s)] \exp \left[-\frac{\beta \Gamma^{\prime}}{4} \int_{0}^{N} \frac{d s}{\vec{r}_{\perp}(s) \cdot \hat{z}}\right]
\end{aligned}
$$

where the integral of $\vec{r}_{\|}(s)$ is absorbed into the normalization constant $\mathcal{N}$. Expand the following integral around small $\delta \vec{r}_{\perp}(s)$ up to quadratic order,

$$
\begin{array}{r}
\int_{0}^{N} \frac{d s}{\vec{r}_{\perp}(s) \cdot \hat{z}}=\frac{N}{l|\hat{t} \cdot \hat{z}|} \log \frac{2 \vec{r}_{\perp \mathrm{c}} \cdot \hat{z}+l|\hat{t} \cdot \hat{z}|}{2 \vec{r}_{\perp \mathrm{c}} \cdot \hat{z}-l|\hat{t} \cdot \hat{z}|} \\
\quad-\int_{0}^{N} d s\left\{\frac{\delta \vec{r}_{\perp}(s) \cdot \hat{z}}{\left(\vec{r}_{\perp \mathrm{c}}\right)^{2}}-\frac{\left(\delta \vec{r}_{\perp}(s) \cdot \hat{z}\right)^{2}}{\left(\vec{r}_{\perp \mathrm{c}}\right)^{3}}\right\}
\end{array}
$$

and then integrate out the variable $\vec{t}(s)$ under the condition that $|\hat{t} \cdot \hat{z}| \ll 1$, the partition function becomes

$$
\begin{aligned}
Z= & \mathcal{N}^{-1} \int_{0}^{\infty} d z_{\mathrm{c}} z_{\mathrm{c}} \exp \left[-N \beta\left(h z_{\mathrm{c}}+\frac{\Gamma^{\prime}}{4 z_{\mathrm{c}}}\right)\right] \\
& \times \int \mathcal{D}[\delta z(s)] \exp \left[\int _ { 0 } ^ { N } d s \left\{-\frac{1}{2 a^{2}}\left(\frac{\partial \delta z(s)}{\partial s}\right)^{2}\right.\right. \\
& \left.\left.-\beta\left(h-\frac{\Gamma^{\prime}}{4 z_{\mathrm{c}}^{2}}\right) \delta z(s)-\frac{\beta \Gamma^{\prime}}{4 z_{\mathrm{c}}^{3}}(\delta z(s))^{2}\right\}\right]
\end{aligned}
$$

Note that $l$ is related to $\vec{t}(s)$ via Eq.(17), and will be integrated out inside the functional integral of $\vec{t}(s)$.

Without the effect from $\vec{t}(s)$ and $\delta \vec{r}_{\perp}(s)$ which expressed in the effective potential of $\delta z(s)$, the polymer acts as a rigid rod. Its equilibrium height is at $z_{\mathrm{c}}=\sqrt{\Gamma^{\prime} / 4 h}$. The ensemble average

$$
\langle\delta z(s)\rangle=\frac{z_{\mathrm{c}}}{2}\left(1-\frac{4 h z_{\mathrm{c}}^{2}}{\Gamma^{\prime}}\right)
$$

The entropic force points upward (downward) when the height of center of mass of the polymer is lower (higher) than $\sqrt{\Gamma^{\prime} / 4 h}$. If $\Gamma^{\prime}=0$ (same dielectric constants), the
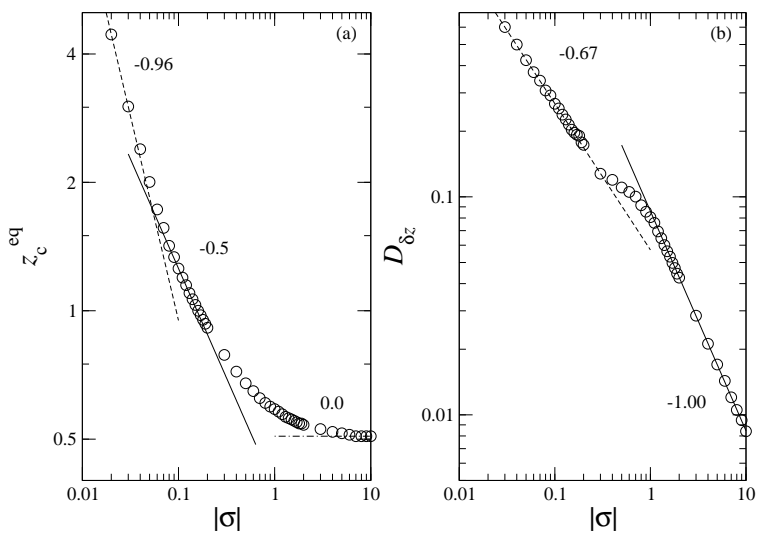

FIG. 2: (a) Simulation results for the equilibrium height of the polymer, $z_{\mathrm{c}}^{\mathrm{eq}}$ (in units of $2 a$ ), as a function of surface charge density, $|\sigma|$ (in units of $q / 4 a^{2}$ ), in logarithmic scale at $\epsilon^{\prime} / \epsilon=0.01$ and $\kappa^{-1}=25$. It shows the scaling exponents decreases continuously from -0.96 (weak compression) to 0 (compression) with the surface charge density. The scaling exponent of -0.5 indicating the crossover (analytically predicted) occurs at $|\sigma| \simeq 0.11$. (b) The thickness of the diffusion layer, $D_{\delta z}$, as a function of surface charge density, $|\sigma|$, in logarithmic scale. It shows the scaling exponents are -0.67 and -1.00 for weak compression and compression, respectively. The crossover occurs at $|\sigma| \simeq 0.11$.

effective potential for $\delta z(s)$ is linear rather than the harmonic. The result for the case of low-dielectric substrate cannot be analytically continued to the case of same dielectric constants. If $\Gamma^{\prime}<0$ (high dielectric substrate), the system is unstable. It implies that the decomposition in Eq.(5) is inadequate in high-dielectric case.

Hence, after integrating out the fluctuation variable $\delta z(s)$ under the ground state dominance (large- $N$ limit), there leaves only the variable $z_{\mathrm{c}}$ in the partition function which determine the effective probability density distribution for the height of the center of mass,

$$
\begin{aligned}
\rho\left(z_{\mathrm{c}}\right)= & z_{\mathrm{c}} \exp \left[-N \beta\left(\left(h z_{\mathrm{c}}+\frac{\Gamma^{\prime}}{4 z_{\mathrm{c}}}\right)\right.\right. \\
& \left.\left.+\frac{z_{\mathrm{c}}^{3}}{\Gamma^{\prime}}\left(h-\frac{\Gamma^{\prime}}{4 z_{\mathrm{c}}^{2}}\right)^{2}-\frac{a}{2}\left(\frac{\Gamma^{\prime}}{2 \beta z_{\mathrm{c}}^{3}}\right)^{\frac{1}{2}}\right)\right]
\end{aligned}
$$

up to a normalization constant. The new equilibrium including the effect from conformational changes is calculated by "force balance", $\partial_{z_{\mathrm{c}}} \log \rho\left(z_{\mathrm{c}}\right)=0$, which gives

$$
h\left(z_{\mathrm{c}}^{\mathrm{eq}}\right)^{2}+\frac{a}{4}\left(\frac{\Gamma^{\prime 3}}{2 \beta\left(z_{\mathrm{c}}^{\mathrm{eq}}\right)^{5}}\right)^{\frac{1}{2}}=\frac{\Gamma^{\prime}}{4}
$$

at large- $N$ limit. For high enough surface charge density that $z_{\mathrm{c}}^{\mathrm{eq}}$ is low, Eq.(13) gives $z_{\mathrm{c}}^{\mathrm{eq}} \sim|\sigma|^{0}$. The polymer is compressed in which the center of mass is independent of the surface charge density. When the surface charge density is lowered such that $z_{\mathrm{c}}^{\mathrm{eq}}$ is high, Eq.(13) reduces to the scaling $z_{\mathrm{c}}^{\mathrm{eq}} \sim|\sigma|^{-1 / 2}$. If the surface charge density is further lowered so that the polymer basically behaves as a colloid (undeformed state), Eq.(12) becomes 
$\rho\left(z_{\mathrm{c}}\right)=\exp \left[-N \beta h z_{\mathrm{c}}\right]$, and hence $z_{\mathrm{c}}^{\mathrm{eq}} \sim|\sigma|^{-1}$. It predicts a continuous crossover from the compressed state to the weakly compressed state. It is different from that obtained by scaling analysis for grafted polymer, predicting a discontinuous jump [15]. Our analytical result is consistent with MC simulation as shown in Fig[2].

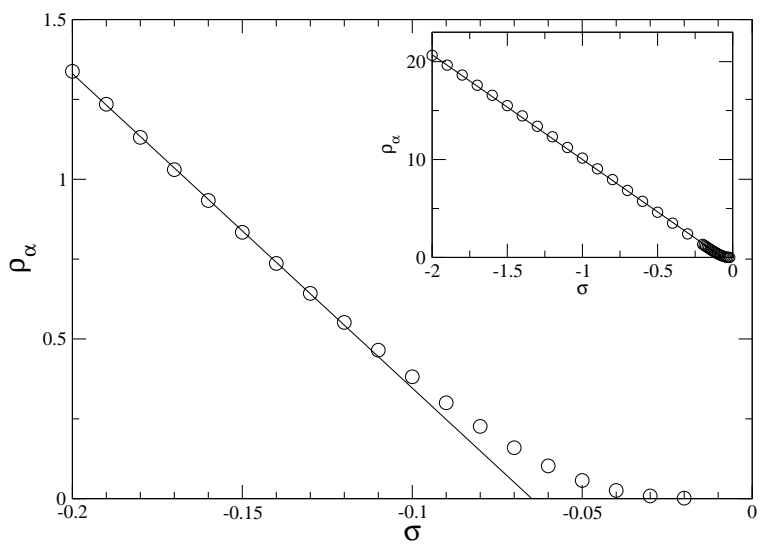

FIG. 3: Monte-Carlo results for the normalized monomer density at the surface, $\rho_{a}$, as a function of surface charge density, $\sigma$ (in units of $q / 4 a^{2}$ ), at $\epsilon^{\prime} / \epsilon=0.01$ and $\kappa^{-1}=25$. The straight line is linearly fit to the data of higher $|\sigma|$. It shows the data starts to deviate from the linearity when $\sigma \simeq-0.11$. Inset: More results to cover a larger range of surface charge density $\sigma$. It shows the data follows the linearity at higher $|\sigma|$.

Besides the position of the center of mass, we also calculate the thickness of the diffusion layer which is defined as the characteristic length scale of the exponential decay of monomer density. We first determine the saddle-point $z_{\mathrm{c}}^{*}$ from Eq. (10) (equivalent to integrating out the variable $z_{\mathrm{c}}$ at large- $N$ limit), which is given by

$$
h\left(z_{\mathrm{c}}^{*}\right)^{3}-\frac{\Gamma^{\prime}}{4} z_{\mathrm{c}}^{*}+\Gamma^{\prime}\langle\delta z(s)\rangle=0
$$

The above equation is then solved self-consistently with Eq. (11) in which $z_{\mathrm{c}}$ is replaced by $z_{\mathrm{c}}^{*}$. The solution is

$$
z_{\mathrm{c}}^{*}=\sqrt{\Gamma^{\prime} / 4 h} \sim|\sigma|^{-\frac{1}{2}}
$$

The effective partition function for $\delta z(s)$ becomes

$$
\begin{aligned}
Z= & \int \mathcal{D}[\delta z(s)] \exp \left[\int _ { 0 } ^ { N } d s \left\{-\frac{1}{2 a^{2}}\left(\frac{\partial \delta z(s)}{\partial s}\right)^{2}\right.\right. \\
& \left.\left.-\frac{\beta \Gamma^{\prime}}{4\left(z_{\mathrm{c}}^{*}\right)^{3}}(\delta z(s))^{2}\right\}\right]
\end{aligned}
$$

which gives the diffusion layer thickness

$$
D_{\delta z} \sim\left(z_{\mathrm{c}}^{*}\right)^{\frac{3}{4}} \sim|\sigma|^{-\frac{3}{8}}
$$

The scaling exponent does not depend on the surface charge density. It means that the local fluctuation $\delta z(s)$ is independent of the polymer conformation, which is consistent with its definition expressed in Eqs. (15)- (6) However, simulation results in Fig 20 show that the scaling exponent is -0.67 , a quite large deviation from our analytical result, -0.375 . The deviation may be due to the approximation of the effective potential up to the quadratic order only. As shown in Fig 2] and 20, both the simulation results of $z_{\mathrm{c}}^{\mathrm{eq}}$ and $D_{\delta z}$ exhibit the crossover between the weak compression and compression regimes occuring at $\sigma \simeq-0.11$.

Finally we also examine the relation between the surface monomer density and surface charge density by MC simulation. Fig 3 shows the simulation data follows the linearity at high enough $|\sigma|$, and start to deviate from the linearity at $\sigma \simeq-0.11$. The linear relation implies the compression regime [13]. The deviation from linearity tells that the polymer starts to be weakly compressed, which is also consistent with the MC results of both $z_{\mathrm{c}}^{\text {eq }}$ and $D_{\delta z}$. Since it is hard to characterize the polymer conformation in MC simulation by the original definition as shown in Fig 1] we would like to propose an alternative (operational) definition for charged polymer adsorption - the linearity between the surface monomer density and the surface charge density.

Support by the National Science Council of the Republic of China is acknowledged under Grant Nos. NSC922816-M-008-0005-6, in part under NSC93-2816-M-0010007-6 (C.H.C.), and NSC93-2112-M-008-014 (P.Y.L.).

* Electronic address: phcch@phys.sinica.edu.tw

[1] Multilayer Thin Films, edited by G. Decher and J.B. Schlenoff (Wiley, 2003), and references therein.

[2] D.H. Napper, Polymeric Stabilization of Colloid Dispersions (Academic, New York, 1984).

[3] A.Y. Grosberg, T.T. Nguyen, and B.I. Shklovskii, Rev. Mod. Phys. 74, 329 (2002), and references therein.

[4] R. Netz and D. Andelman, Phys. Rep. 380, 1 (2003), and references therein.

[5] F.W. Wiegel, J. Phys. A 10, 299 (1977).

[6] M. Muthukumar, J. Chem. Phys. 86, 7230 (1987).

[7] R. Varoqui, A. Johner, and A. Elaissari, J. Chem. Phys. 94, 6873 (1991).

[8] R. Varoqui, J. Phys. II 3, 1097 (1993).

[9] I. Borkuhov, D. Andelman, and H. Orland, Europhys. Lett. 32, 499 (1995).

[10] X. Chatellier and J.F. Joanny, J. Phys. II 6, 1669 (1996).

[11] A.V. Dobrynin, A. Deshkovski, and M. Rubinstein, Phys. Rev. Lett. 84, 3101 (2000).

[12] A.V. Dobrynin and M. Rubinstein, J. Phys. Chem. B 107, 8260 (2003).

[13] C.H. Cheng and P.Y. Lai, Phys. Rev. E 70, 061805 (2004).

[14] C.H. Cheng and P.Y. Lai, cond-mat/0403722

[15] O.V. Borisov, E.B. Zhulina, and T.M. Birshtein, J. Phys. II 4, 913 (1994).

[16] V. Yamakov et al., J. Phys.: Condens. Matter 11, 9907 (1999). 
[17] R.R. Netz and J.F. Joanny, Macromolecules 32, 9013 (1999).

[18] R. Messina, Phys. Rev. E 70, 051802 (2004). 